\title{
Tadpole VR - Virtual Reality Visualization of a Simulated Tadpole Spinal Cord
}

\author{
Marius N Varga, Robert Merrison-Hort, Paul Watson, Roman Borisyuk and Dan Livingstone
}

\begin{abstract}
Recent advances in "developmental" approach (combining experimental study with computational modelling) of neural networks produces increasingly large data sets, in both complexity and size. This poses a significant challenge in analyzing, visualizing and understanding not only the spatial structure but also the behavior of such networks. This paper describes a Virtual Reality application for visualization of two biologically accurate computational models that model the anatomical structure of a neural network comprised of 1,500 neurons and over 80,000 connections. The visualization enables a user to observe the complex spatio-temporal interplay between seven unique types of neurons culminating in an observable swimming pattern. We present a detailed description of the design approach for the virtual environment, based on a set of initial requirements, followed up by the implementation and optimization steps. Lastly, the results of a pilot usability study are being presented on how confident participants are in their ability to understand how the alternating firing pattern between the two sides of the tadpole's body generate swimming motion.
\end{abstract}

Key Words - Data visualization, Neuroscience, Virtual Reality, Visualization, Scientific Visualization, Immersive

- M.N. Varga

Affiliation: University of Plymouth, School of Computing and Mathematics, Drake Circus, Plymouth, PL4 8AA, $U K$.

- E-mail: marius.varga@plymouth.ac.uk

- Orchid ID: 0000-0001-7655-9829

- B. Merrison-Hort

- Affiliation: University of Plymouth, School of Computing and Mathematics, Drake Circus, Plymouth, PL4 8AA, $U K$.

- E-mail: robert.merrison@postgrad.plymouth.ac.uk

- Orchid ID: 0000-0001-8215-7527

- P. Watson

- Affiliation: University of Plymouth, School of Computing and Mathematics, Drake Circus, Plymouth, PL4 8AA, $U K$.

- $\quad$ E-mail: paul.watson-1@plymouth.ac.uk

- Orchid ID: 0000-0003-2797-0026

- R. Borisyuk

- Affiliation: University of Exeter, College of Engineering, Mathematics and Physical Sciences, Harrison Building, Streatham Campus, North Park Road, Exeter EX4 4QF, UK and Institute of Mathematical Problems of Biology, The Branch of Keldysh Institute of Applied Mathematics of Russian Academy of Sciences, Pushchino, 142290, Russia

- Email:r.m.borisyuk@exeter.ac.uk

- $\quad$ Orchid ID: 0000-0003-1384-9057

- D. Livingstone

- Affiliation: University of Plymouth, School of Computing and Mathematics, Drake Circus, Plymouth, PL4 8AA $U K$.

E-mail: D.Livingstone@plymouth.ac.uk

- $\quad$ Phone: +44(0)1752 586250

- Orchid ID: 0000-0001-8211-886X

\section{ACKNOWLEDGMENT}


The authors would like to thank everyone who contributed to this paper. Special thanks to the Interactive Systems Studio team for constant support, especially to Luke Angell. Great thanks to Professor Michael Thrasher for lending his voice to the visualization. We are grateful to Professor Alan Roberts and Dr. Stephen R. Soffe from Biological Sciences - University of Bristol for their valuable advice and feedback about the visualization. More details about the project is available here: http://iss.io/tadpole/, including links to two 360 videos that can be viewed through a stereoscopic headset for mobile phones.

\section{INTRODUCTION}

At 2 days old, young tadpoles do not have their brain fully developed, yet they are sensitive to touch, pressure, light intensity and water currents. They can flex, swim away, change direction, speed up, slow down, and struggle when grasped. Research by (Roberts et al., 2010) attributed this behavior to seven types of neurons in the hatchling's spinal cord. A simple touch on the side of the tadpole will initiate a firing sequence inside the spinal cord's neural network that culminates in an alternate swimming pattern firing along the spinal cord. The spinal cord neural network arrangement and the interplay of its 7 unique neuron types enables the tadpole to generate an alternating swimming pattern, independent of its cognitive ability.

Visualization of such spatio-temporal processes, e.g. simultaneous propagation of multiple spikes in 3D space is a challenging problem (Ferrario et al., 2018). A biological neural network is composed of individual neurons that fire at different times (Marder et al., 2015). The complex firing pattern (spiking) is a combination of individual neurons spiking (microscopic level) and complex visual structures generated by all the spiking neurons (macroscopic level). Therefore, individual position of the neurons and the neural network connectivity are crucial in observing and understanding the relationship between the spatio-temporal spiking pattern and behavior output. Using traditional graph visualization techniques is nearly impossible to observe this complex interplay.

We believe that using an immersive Virtual Reality (VR) environment that places the user amongst the data, rather than looking at it, enables the user to gain a better overview of the spatio-temporal arrangement. (Bryson, 1995), describes VR as “...the use of various computer graphics systems in combination with various display and interface devices to provide the effect of immersion in an interactive three-dimensional computer-generated environment in which the virtual objects have spatial presence." Therefore, the main goal of this application is to enable users to gain an overview and understanding of a relatively complex neuronal interplay dynamic that presents a challenge even for seasoned neuroscientists due to its sheer volume and complexity (see Fig. 1).

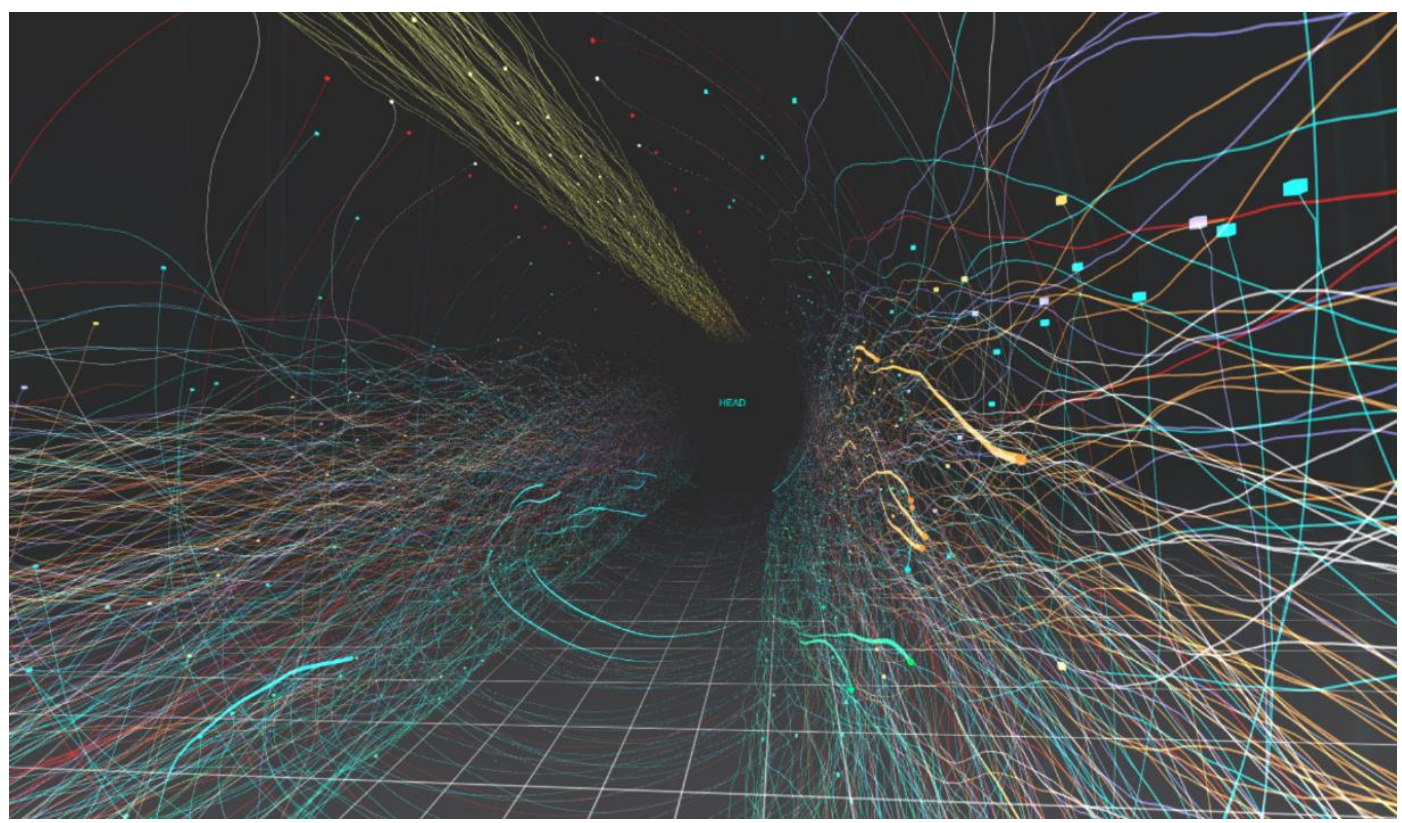

Fig. 1 Image capturing the complex structure of a simulated neural network with 1,500 neurons and over 80,000 connections. Cubes represent neurons and the colored lines represent axons (connections between neurons).

In this paper, we describe the approach and the development process for Tadpole VR. Section 2 of the paper draws a parallel to existing approaches in visualising 3-dimensional complex neuronal structures in VR and we 
describe the 2 biologically accurate computational models (anatomical model and physiological model) that generated the data and their mutual interdependence.

(Bryce S, 1996) suggests that a top-down approach (design the application through requirements) combined with a bottom-up approach (implementation based on the hardware and software capabilities), is the key to a successful VR application. Section 3 unpacks the design and implementation of the Tadpole VR application following Bryce's model. Section 3 is divided in two parts, first part explains the themes/metaphors (Bryce S, 1996) used for describing the virtual environment and its interaction metaphors. Second part depicts the bottomup approach; implementation and technical challenges encountered in rendering 1,500 neurons and over 80,000 connections.

Section 4 is dedicated to a formative pilot usability study to examine participant's ability to observe and understand the process of generating swimming activity (the alternating firing pattern between two sides of the tadpole body) from a simple touch on the side of a tadpole. Section 4 starts with the methodology employed, moves to analysis and culminates in the results section. The result section covers findings and gives details of various statistical tests carried out in order to understand their significance.

Section 5 focuses on Discussion and unpacks the implication of the findings in the usability study. It is followed up by potential applications for this approach and finally concludes the manuscript with the Conclusion and Further Work section that covers future directions.

\section{BACKGROUND AND RELATED WORK}

\subsection{Background on computational models}

The nervous systems of animals and humans can be considered as large and complex networks of interconnected cells (neurons). These neurons communicate with each other via short ( $5 \mathrm{~ms})$ electrical pulses ("spikes"). Incoming spikes can either raise (excite) or lower (inhibit) the voltage of a neuron, if enough excitatory input is received then the neuron may itself generate a spike. One of the fundamental questions in neuroscience relates to the relationship between the structure of neuronal connectivity (how neurons connect to each other) and the functionality of the network (the spiking activity based on the neuronal connections). This question has proven to be quite challenging because of the difficulty in capturing electrophysiological recordings at such a small scale. The solution is to create computational models of biological neural networks that enable neuroscientists to measure and analyse firing neurons.

\section{Anatomical model}

In their paper, (Borisyuk et al., 2014) describe a new computational method (“developmental approach") that can generate complete biologically accurate neural networks ("connectomes") that represent the spinal cord of a 48-hour old and $5 \mathrm{~mm}$ long hatchling Xenopus tadpoles. The tadpole is simple enough for anatomical and electrophysiological studies yet neurologically complex enough to allow interesting discoveries that could be applicable to organisms that are more complex.

The main idea behind the developmental approach is to simulate the process of neuron growth with connections appearing when growing axons intersect other neurons. Thus, the connections are not prescribed but appear as result of model simulation. The anatomical model includes approximately 1,500 neurons of seven different types, split across left and right body sides and over 80,000 connections between various neurons. Anatomical model generates information for neurons and their corresponding axons. Two of the generated files are being used in this VR application:

First file contains information regarding neurons:

- Neuron's ID

- Neuron's side of the body (left or right)

- Type of neuron: 7 different types of neurons (RB, dlc, dla, dIN, cIN, aIN, mn) (see details in Borisyuk et al., 2014)

- Neuron's position in the rostro-caudal (head-to-tail) direction measured in micrometers. The axon's origin point.

Second file contains information about the axons for each neuron:

- Neuron's ID and type (this gives us the link to the Neuron's location).

- A set of pairs of numbers, one for each point along the axon, in which the first number is the rostrocaudal (head-to-tail) co-ordinate and the second is dorso-ventral (up-down). Both numbers represent values in micrometers. The dorso-ventral coordinates represent neuron's location are always relative to 
the side of the body (left or right), so a positive value means the same side of the body and a negative value means the opposite side of the body.

Note: Most neurons have a primary and a secondary axon, but for this model only the primary axons are used as they provide enough information to observe the firing pattern while it avoids cluttering the view of the secondary axons.

\section{Physiological model}

Once the anatomical models have been generated, a different computational model, "physiological model" (Roberts et al., 2014) is used to simulate the electrical activity in the network. Physiological model generates a file that contains a list of spikes fired by the individual neurons during the simulation:

- Time when the spike occurred, the value measurement is in milliseconds; the simulation starts at zero.

- Neuron's ID - neuron that generated the spike.

Throughout this paper, the term "anatomical model" refers to the model developed by (Borisyuk et al., 2014) and "physiological model" refers to the computational model developed to generate the spiking activity.

\subsection{Related Work}

Visualization of biological neural networks for small creatures has been done before, (Bruckner et al., 2009) developed BrainGazer - visualization of a fruit fly brain, using a photorealistic rendering. Their main focus was on the spatial rendering of pathways as they reveal the spatial relationship between various neuronal clusters.

A similar approach on cluster visualization was attempted on a more complex neuronal system, human connectome by (Lin et al., 2011), (Xia et al., 2013) and (Sherif et al., 2015). All these applications capture the functionality of human connectome clusters but with a strong focus on analysis, enabling the user to query, select and trace the neuronal clusters. These approaches have one thing in common, the use of a two-dimensional screen to render a three-dimensional object. The interaction and navigation of these systems is achieved through mouse and keyboard, making it less intuitive than a three-dimensional interaction with an object, in a VR virtual environment.

In their paper, (Arsiwalla et al., 2015) use a more intuitive interaction metaphor, by placing the user in an immersive environment (CAVE) and using hand recognition software that allows the user to interact without any additional peripherals. Their approach enables the visualization of a large-scale simulation of neuronal dynamics in the human brain with separate unique projections on each wall in order to enable the user to get a better understanding of the neural network.

All the systems mentioned so far capture the structure of the neuronal layout, but the temporal aspect has been limited to interaction. The temporal aspect of the visualization is captured through querying the state of various neuronal clusters at different points in time. In their paper, (Kapri et al., 2011) approach was to capture the activity flow between various brain areas by using a CAVE system with a stereoscopic projection. Their focus was on the temporal aspect of a neuronal network firing. The intent was to capture neurons firing at both macroscopic and microscopic level. (Kapri et al., 2011) suggested that a neural network visualization "... should convey the influence of the macroscopic communication (between different brain areas) on the interaction at a microscopic level (between individual neurons).". The result is an established example, where the relationship between the cells interacting at both microscopic and at macroscopic level is significant in understanding the neuronal interaction. Even though the neuronal activity is easily observable due to the stereoscopic effect and sense of depth in a VR environment, its impact is limited by the spatial arrangement of the neurons. Placement of neurons in a grid-like configuration removes the spatial aspect of a neural network, focusing only on the temporal aspect of it. Tadpole VR uses both, the spatial layout of the neurons and the temporal pattern generated by the spiking neurons. This approach enables the user to gain a better understanding of the complexities of a functioning neural network.

Although (Kapri et al., 2011) application was successful in capturing the temporal element of the firing neural network it was still focusing on small sections of the brain, not showing a full network connectivity. In NeuVis implementation, (Marks, 2017) approach was to use a spatio-temporal capture of an entire spiking neural network. NeuVis is a visualization extension to (Kasabov et al., 2016) - NeuCube learning environment. NeuVis enables the user to navigate the virtual environment and enables the user to control the playback of the spiking neurons. Tadpole VR and NeuVis are closely related from a VR implementation point of view. Tadpole VR also uses controls to trigger the neurons firing sequences and the user can explore the virtual environment. The difference is in the number of neurons and axon connections Tadpole VR is capable of rendering. NeuVis renders 1,500 neurons and over 15,000 connections while Tadpole VR renders 1,500 and over 80,000 connections. This a significant increase in three-dimensional objects rendered inside the virtual environment while still maintaining a 
consistent 75 frames per second.

\section{VR SYSTEM OF THE TADPOLE SPINAL CORD}

One of the main drivers for development of this application is the difficulty to gain an overview on how the anatomical and the physiological models work together, due to the sheer scale and complexity of the data. In Fig. 2 an output in two-dimensional format of the resulting data from the anatomical model representing individual connections between various neurons, displayed. This approach clearly does not work, as connections between various types of neurons are not clear. Furthermore, overlaying the physiological data on top of the existing representation will clutter the view even further.

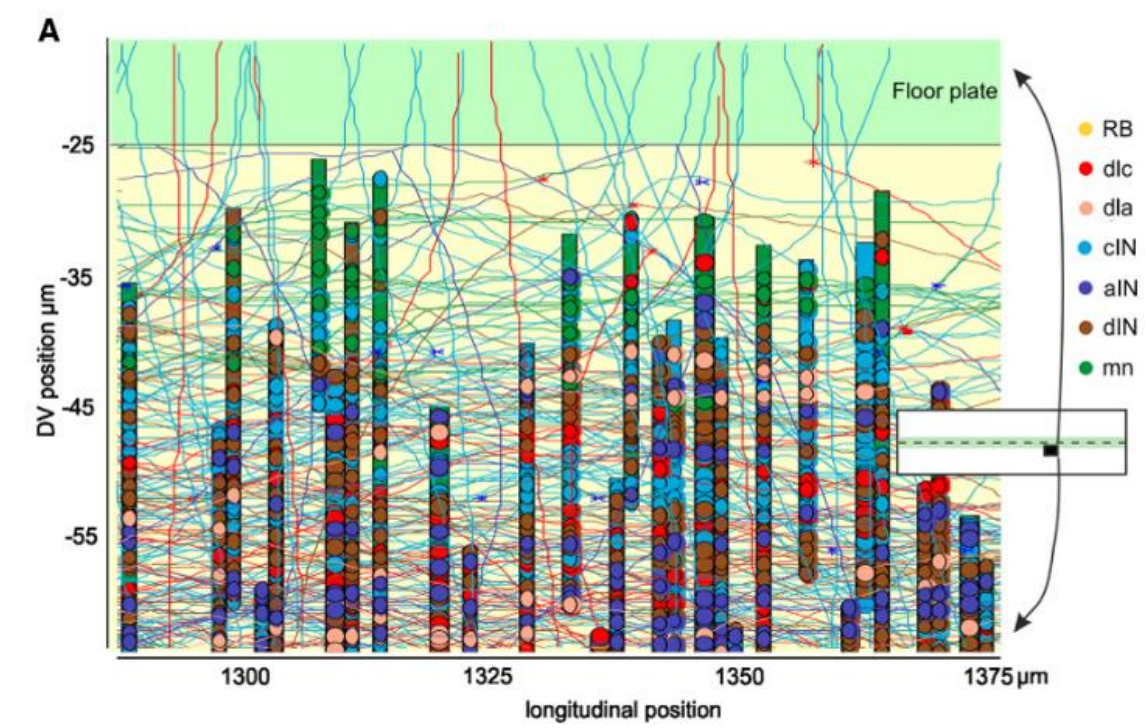

Fig. 2 The original graph generated from the growth model described in the (Borisyuk et al., 2014). Seven distinct neurons but due to the high number of axon connections, it is very difficult to identify individual neurons and their connections. This image is reproduced with authors' permission.

\subsection{Requirements}

In his paper "Approaches to the Successful Design and Implementation of VR Applications”, (Bryson, 1995) defines a virtual environment as an interactive three-dimensional computer-generated environment. He argues, a successful VR application needs a theme also known as a metaphor. A metaphor is a way the user is supposed to relate to the virtual environment. For this application, the scale of the user is reduced (shrank) in order to allow them to fly inside the neural network of the Xenopus tadpole. An audio recording conveys this metaphor to the user before they enter the neural network, which sets the expectations.

From a design point of view, a "top-down" approach is employed focusing mainly on 3 areas: Overall environment, Information presentation and Interaction. As a result, we compiled a list of requirements that enables the user to gain and understanding and overview of the spatio-temporal dynamics of the neural network:

Stages - The information visualized should be delivered in stages in order to enable the user to gain an understanding of the individual chunks of information and not to get overwhelmed by a huge wave of information delivered in one-step. Ideally, the user would trigger each stage, and would have full control of the application's pace.

Neuronal Spatial Placement - The individual placement of each neuron and the shape of the spinal cord should be observable. Shape of the firing pattern is crucial in understanding how the firing pattern propagates along the rostro-caudal (head to tail) direction on the tadpole's body.

Neurons type and roles - The user should be able to gain an understanding of each of the 7 different types of neurons. The focus should be on the neuron's role, unique spatial positioning and the overall interplay, as a combination of these three elements results in the alternate swimming pattern.

Microscopic and Macroscopic view - The user should be able to switch relatively seamlessly between the 
microscopic view of the network (looking at individual firing neurons) and macroscopic view (visual patterns created by the temporal spiking of multiple neurons).

Visual swimming pattern - The user's main goal in using this application is to observe the alternate swimming pattern generated by the spiking neurons.

\subsection{Top-down design}

\section{Stages}

Before revealing the spiking neural network to a user, we ensure the user is comfortable inside a virtual environment and familiar with the interaction metaphor. Therefore, the application has 3 distinct stages:

First stage - familiarizes the user with the controls and the interaction method with buttons inside the virtual environment.

Second stage - introduces the user to a visually rich virtual environment in order to minimize the novelty factor of using VR and to introduce the metaphor used for the visualization.

Third stage -visualizes the neural network and the spiking neurons. This stage divided further into sub-stages and the user triggers each sub-stage inside the virtual environment.

First stage - The user starts inside a virtual environment with one single button available, in order to familiarize the user with the interaction metaphor (pressing the button inside the virtual environment). User's perspective inside the virtual environment is first person. Through this perspective a ray-casting selection technique (Lee et al., 2003) is used, combined with a trigger of a controller in order to interact with any buttons inside the virtual environment. The ray-cast is represented by a "reticle" (sprite) in the center of the screen, in a shape of a small red circle, which upon collision with an interactive element inside the virtual world it snaps at the collision point and highlights the interactable element.

For every interaction within the virtual environment, associated text provides details about the current interactive element (See Fig. 3). In addition, a voice over system that conveys information to the user in order to guide them to the next stage or to provide additional information for a particular point of interest. The voice over system uses a pre-recorded set of messages allocated based on the location and the time of the visualization. This helps the user to understand the next stage in the visualization. The user has the option to re-play audio for more complicate topics related through the voice-over system.

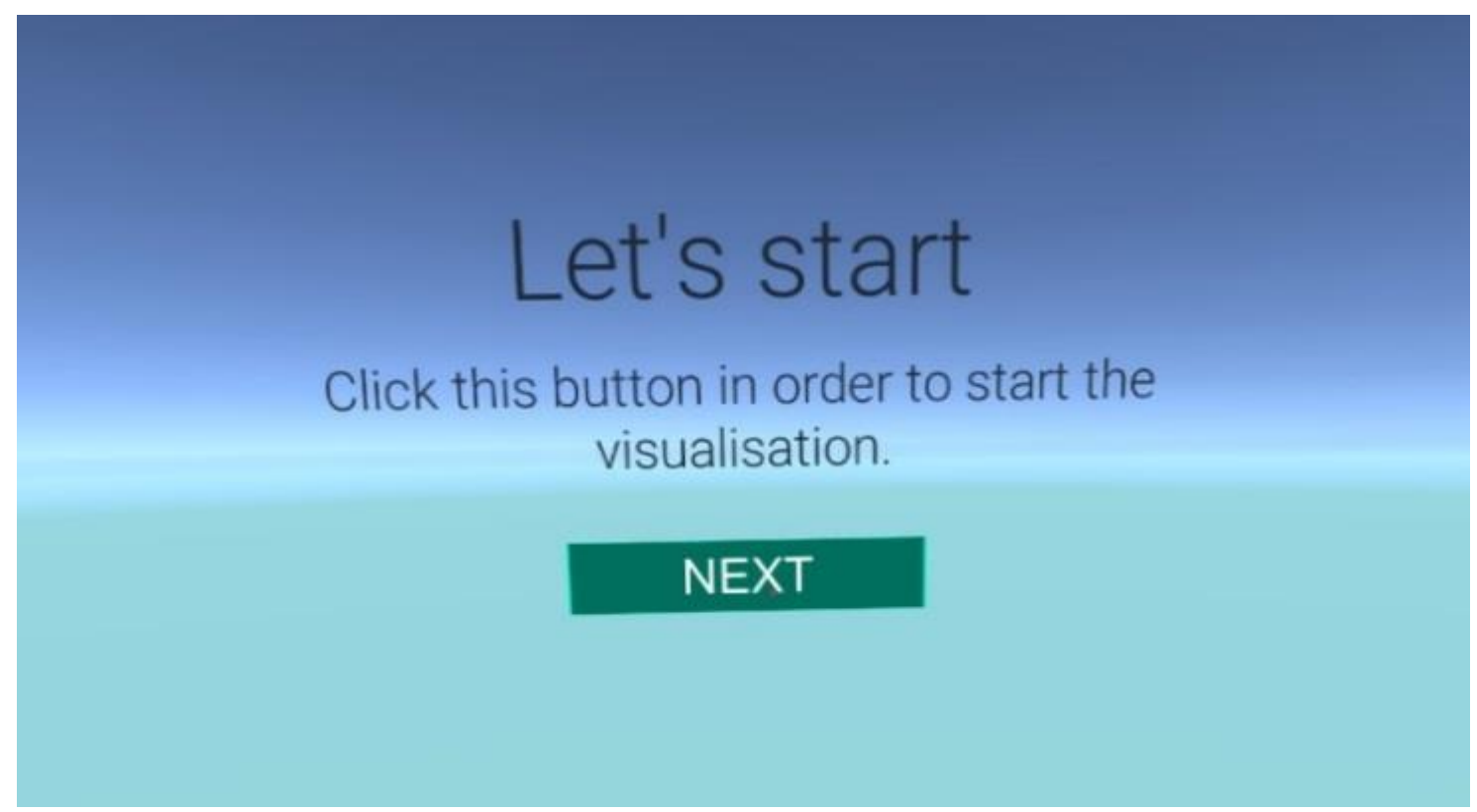

Fig. 3 The initial interactive element (button) used in the visualization. This is part of the first stage where the user learns how to interact with the buttons and how to follow instructions coming from the voice over system.

Second stage - The second stage introduces the user the virtual environment, in order to acclimatize them to the novelty factor brought by the VR experience. Some users might have never experienced a VR environment and might find it difficult to focus on a task until the novelty of the VR experience wears off. The user is exposed to a realistically rendered environment, looking at a pond in the middle of a forest surrounded by vegetation and rocks (See Fig. 4). Photogrammetry technique (Esmaeili \& Thwaites, 2016) is used; which is a process where 
multiple photos of a real object are taken in situ, mapping its entire surface. These photos are collated with a roughly 10 to 20 degrees angle apart. Through Agisoft Photoscan ${ }^{1}$, these images build a very high-resolution $3 \mathrm{D}$ model and its supporting textures. This model is optimized through $3 \mathrm{D}$ content creation packages such as $3 \mathrm{ds}$ $\mathrm{Max}^{2}$ in to reduce the number of polygons and improve the performance of the final scene. The detail from the high-resolution models is "baked" to form a series of texture maps that display these details onto optimized lowresolution models. The final output is a realistic 3D asset that captures life-like detail found through photography and runs 75 FPS in the VR application.

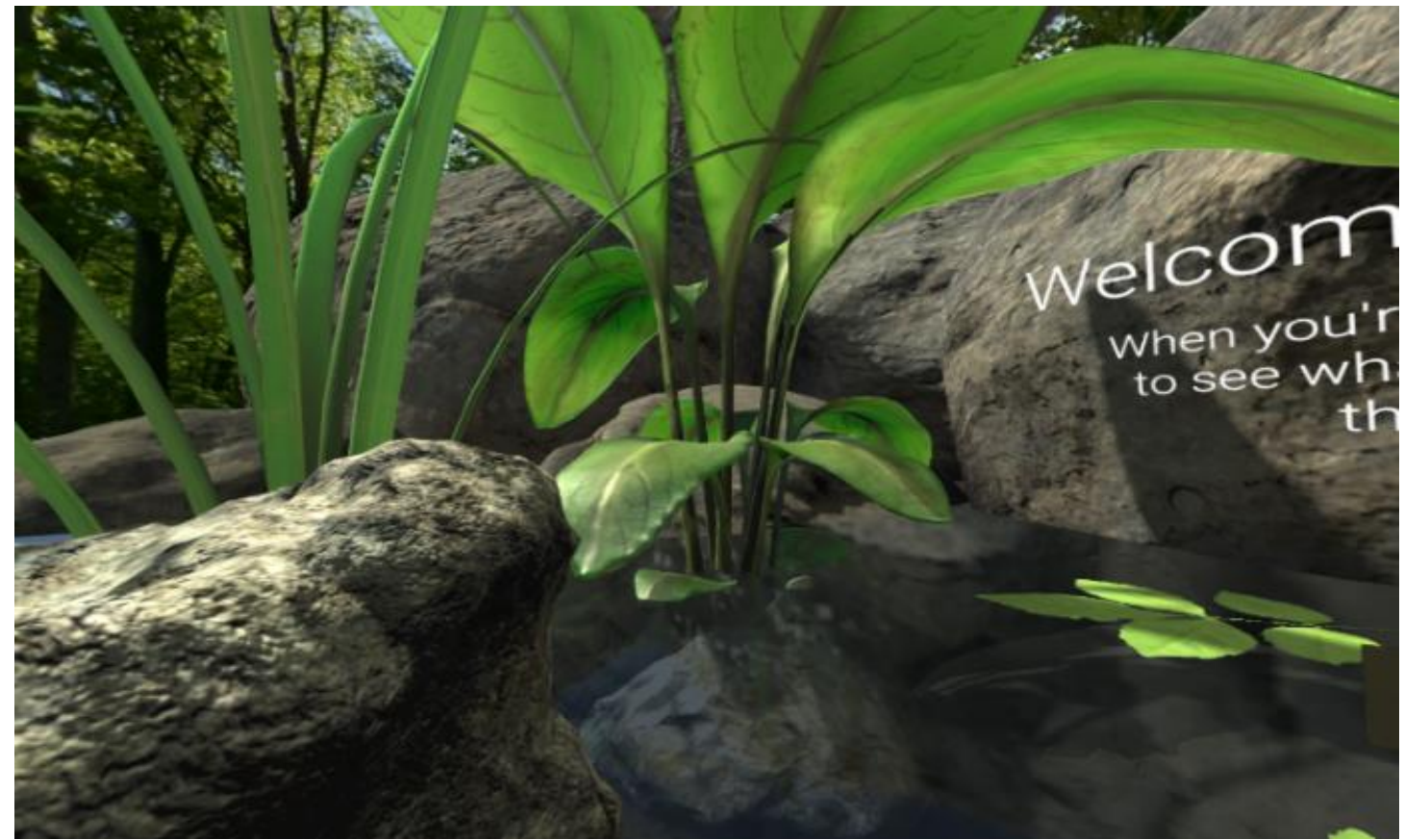

Fig. 4 Screen shot of the second stage of the visualization. In the second stage, the user is going through an acclimatization process inside the VR environment, in order to experience the stereoscopic view without distractions.

In order to prepare the user for the next stage, a small tadpole is introduced, swimming in the middle of the pond. The tadpole is the interactive object and clicking on it loads the third stage. The interactive tadpole is accompanied by audio, which describe the metaphor for the stage 3. The metaphor is to reduce the size of the user to neuronal scale and placing it inside a tadpole' spinal cord to observe the firing neural network.

Third stage - The third stage is the actual visualization of the neural network and we believe just like (Kapri, 2011) that the user will build a better mental model of the network if the application enables exploration inside the virtual environment. At the beginning of the third stage, the user will familiarize with the locomotion inside the VR space by going through a set of training steps. The training is in the form of audio prompts supported by text floating in three-dimensional space that guide the user to move around the virtual environment. The training system is waiting for the user to execute the assigned task and on completion moves onto the next step, until the training is complete.

\section{Locomotion}

The flying model implements the locomotion inside the virtual environment as described by (Ware, 2012b). The user moves forward, backwards, and side-to-side and it can rotate the character. A better way to understand the controls is if we imagine the user is controlling the body of a virtual character with the controller, while the HMD controls the head of the character. The controls do not allow the user to move directly vertically, but in order to go up or down a combination of the rotation angle of the HMD on x-axis (the pitch) and the forward movement vector enables the user to move up or down. This allows the character to move forward and up if the user is looking up or forward and down if the user is looking down. Limited number of users reported simulator sickness

\footnotetext{
1 Agisoft Photoscan - http://www.agisoft.com

2 3D Studio Max - http://www.autodesk.co.uk/products/3ds-max/overview
} 
(Kolasinski, 1995) when they travelled forward while looking sideways. This situation can create issues due to the feedback imbalance between inner ear and the visual feedback perceived. Restricting the ability to travel only in the direction the user is looking should ameliorate the problem, implementation and testing is needed though.

\section{Goals location}

User positioning inside the virtual space is crucial as they might miss important firing sequences by exploring the virtual environment. In order to make sure the user is present at the point of interest, interactive objects (buttons) reside at that particular location. The role of the buttons is to trigger the next step in the visualization sequence. The buttons interactivity is limited to a small distance; therefore, the user needs to be close to the button to trigger the next step, ensuring proximity to points of interest.

In order to help the user with orientation and navigation two sets of arrows guide the user inside the virtual space. First set of arrows is a directional arrow forming a straight line from the user's location to the location of the next important step in the visualization (See Fig. 5, aqua colored arrows). This allows the user to move from their current position to the next relevant location. The second set of arrows are semi-transparent curved arrows, which help the user with orientation (See Fig. 5, pink colored arrows). The circular arrows come into view when the user is not facing the next point of interest guiding the user to rotate left or right in order to face the point of interest.

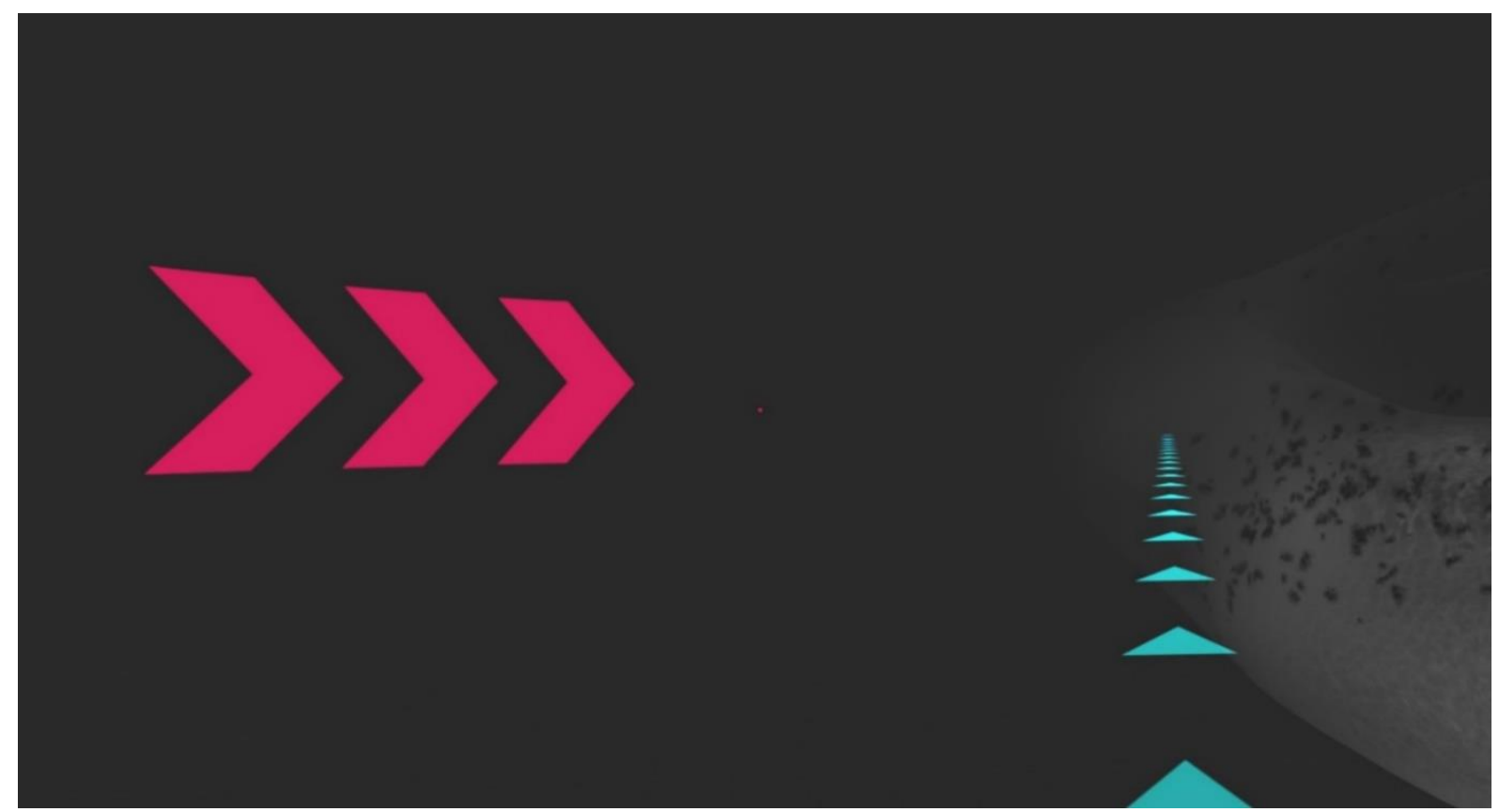

Fig. 5 Image depicting two sets of arrows used for navigation. The blue arrows are the directional arrows that form a straight line from the virtual character position to the objective. The second set of arrows (pink arrows) are orientation arrows, they help the user turn towards the objective, even though the objective is not visible.

\subsection{Building the neural network}

The growth model generates the entire neural network with two-dimensional values ( $\mathrm{x}$ and $\mathrm{y})$. $\mathrm{x}$ corresponds to a position along the rostro-caudal direction and y corresponds to dorso-ventral direction. On the dorso-ventral (up-down) direction, positive values occupy the top part, value of zero reside in the middle and negative values cover the bottom part (see Fig.6 for details). (Borisyuk et al., 2014) considers the thickness of the neural wall as insignificant in comparison to rostro-caudal (head-to-tail) values and dorso-ventral (top to bottom) values, so the $\mathrm{z}$ value is not calculated.

The main requirement to render neurons in a three-dimensional environment is to transform the data from twodimensional to three-dimensional coordinates. The tadpole's biological spinal cord has an approximately cylindrical shape; in order to match this shape, we turn the flat shape of the model in Fig. 6 into a cylinder. For that, we need to apply a small mental exercise in order to understand the approach. Imagine the layout in Fig. 6 as a sheet of paper. Joining the top and bottom sides (the yellow areas called RB column), the flat piece of paper becomes a cylindrical shape. Fig. 7 shows the resulting shape with the dotted line (dorsal midline) representing 
the two sides that have been joined together. "Folding" the 2D model environment in Fig.6 into a cylinder, keeps unchanged the value for $\mathrm{x}$ (rostro-caudal) coordinates but for $\mathrm{y}$ (dorso-ventral) coordinates a new set of values need to be generated for $y$ and axis.

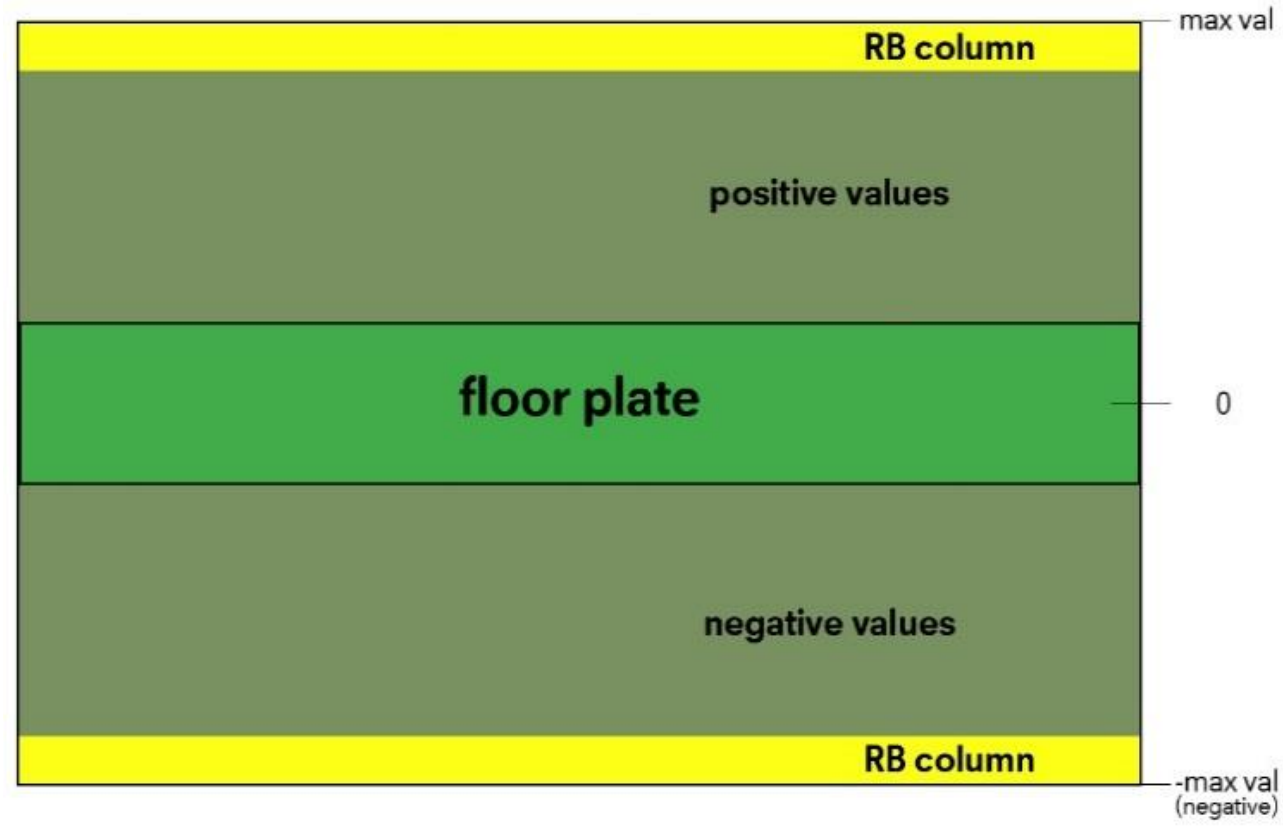

Fig. 6 This diagram represents the spread of values inside the anatomical model. The green area is the relative center with a value of zero. Positive values are above it and negative values are below it. Turning this layout into a cylinder shape places the green area "floor plate" at the bottom of the cylinder and the two yellow areas "RB column", will join together at the top of the cylinder

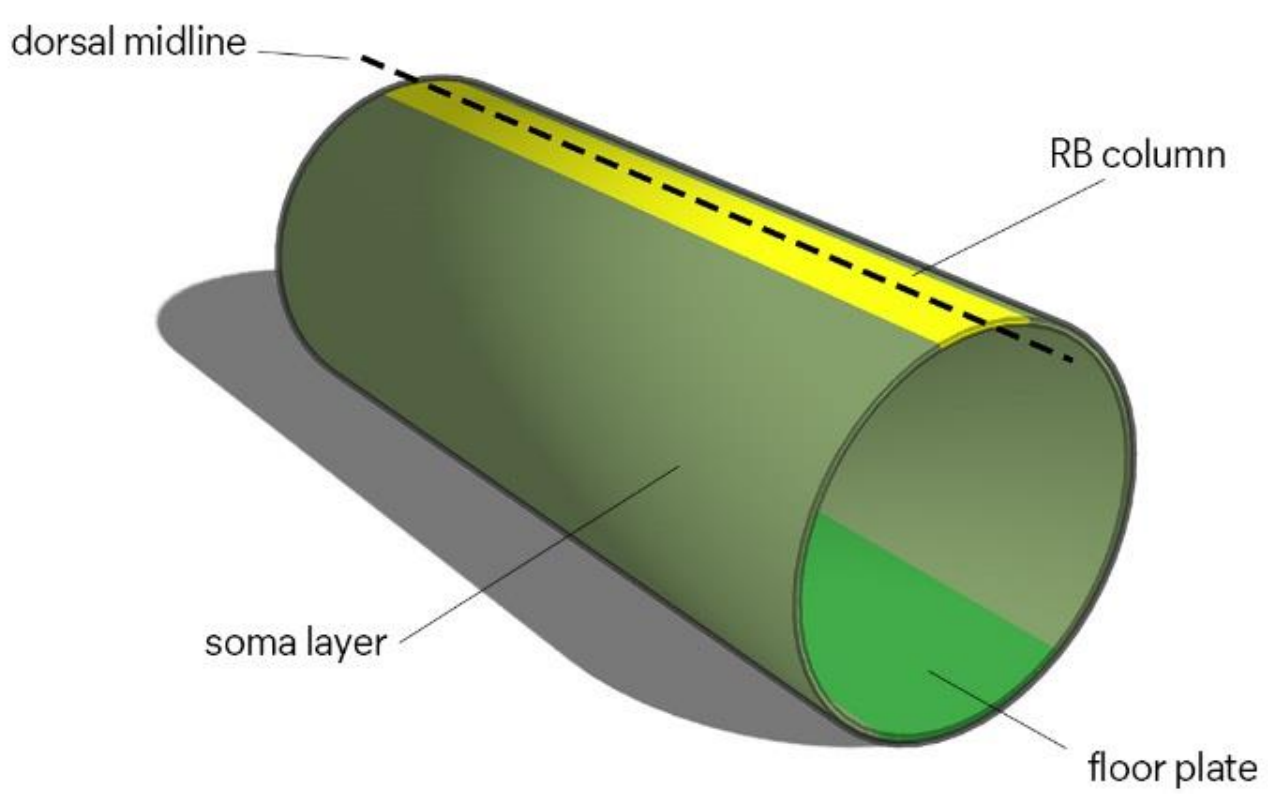

Fig. 7 Diagram representing the layout of the values in the anatomical model after transformation of the layout into a cylindrical shape. The floor plate is at the bottom and the two RB columns connect along the dotted line (dorsal midline). Please check Fig. 6 for the initial shape of the layout. 
Below, a set of steps present the transformation the $\mathrm{y}$ value of each neuron in the data set to a $\mathrm{y}$ and $\mathrm{z}$ value that maps in a three-dimensional environment:

1. Identify the highest value for $y$ (maxVal) out of the entire dataset and set maxVal as the diameter of the cylinder. From the diameter, deduct cylinder's radius, which is half of the diameter.

2. Calculate an angle (ang) in degrees for each y (position.y) value from the original data. The angle needs to go from 0 to 360 degrees so any positive values (from the original data set) go from 0 to 180 . The pseudo code below explains the logic:

ang $=180-(($ position.y /_maxVal $) * 180)$;

Any negative values (from the original data set) will go from 180 to 360 using this pseudo code:

ang $=180+\left(\left(\right.\right.$ position.y $/ \_$maxVal $\left.) * 180\right)$

3. Using the angle, generate a new $y$ value and a new $z$ value. The $x$ value for rendering is identical to $x$ from the original data and for $\mathrm{y}$ is cosine of the angle and for $\mathrm{z}$ is sine of the angle:

pos.x $=\_$position. $x$

pos.y $=$ radius $* \operatorname{Cos}($ ang $)$

pos. $z=$ radius $* \operatorname{Sin}($ ang $)$;

For this visualization, we assumed the thickness of the spinal cord wall at 10 microns, after a consultation with the authors of the anatomical model. In order to generate the thickness of the wall, a uniformly distributed random variable has been added for the $\mathrm{z}$ values of each neuron. This approach accurately represents the relative positions of neurons and axons side of 10-microns thick wall of the cylinder without having an impact in the functionality of the neural network.

The same approach has been applied to generate rendering data for the primary axon, which is the connecting element between various neurons. Each axon has a set of points that define its position in the virtual environment. Number of points for each axon varies based on the information received from the data file. Once the individual three-dimensional values for each point of each axon has been generated, the resulting data is being used to create a path to propagate a visual spike along the axon based on the timing generated by the physiological model.

The spiking data is measured in milliseconds but for visualization's purposes that will be too fast, the users will not be able to spot the swimming pattern. Based on the feedback received during the development phase we transformed the times from milliseconds to seconds (slowing down the time in order to enable the user to follow the firing patterns).

\subsection{Bottom-up - Rendering and Optimization}

\section{Hardware}

As (Bryson, 1995) has suggested, a top-down approach in designing a virtual environment, typically needs to be reconciled by a bottom-up designed imposed by the limitation of the hardware and the software used. Some of the design compromises made in this application were due to the balance needed between the two approaches.

In recent years, Unity3 $\mathrm{D}^{3}$ ("Unity" for short) has become one of the most popular development tools for 3D interactive content. Its deployment versatility and scripting support has made it not only suitable for game development, but also for research driven applications (Moran et al., 2015), (Hubbell and Kepner, 2012). Additional support for stereoscopic headset displays is being provided through software development kits (SDKs) and consistent feature updates has made Unity3D 4.4.2 the preferred tool for developing this VR visualization.

For a VR headset, Oculus Rift Development Kit 2 (“DK2") head mounted display (HMD) built by Oculus 4 , has been used. DK2 was released in July 2014 and at the time of development DK2 had considerable Unity support and integration (Yao et al., 2015) and was a significant improvement from its predecessor ("DK1"). DK2 uses a low persistence OLED display with a resolution of $1920 \times 1080$ (960 × 1080 per eye), with a $75 \mathrm{~Hz}$ refresh rate, a 100 degrees nominal field of view, 6 degrees of freedom and a latency of $\sim 30 \mathrm{~ms}$. For rotational tracking DK2

\footnotetext{
${ }^{3}$ Unity - Scripting API: Mesh - https://docs.unity3d.com/ScriptReference/Mesh.html

${ }^{4}$ Oculus - https://www.oculus.com/
} 
uses a Gyroscope, Accelerometer and Magnetometer. For positional tracking the HMD uses a separate camera "near infrared" sensor with a tracking volume of 72 degrees horizontal tracking and 52 degrees vertical tracking.

XBOX360 controller has been used for interaction or movement inside the virtual environment. Although the controller was dedicated to the XBOX 360 gaming console, it had extensive support for Windows environment and natively supported by Unity. For navigating in the virtual world, the directional joysticks have been used and for interacting with elements in the virtual world the "Right trigger" on the XBOX controller.

\section{Rendering}

Commonly, in VR visualizations, individual data elements are represented by shaded spheres (Marks, 2017), (Kapri et al., 2011) or stylized spheres (Marks et al., 2014). For this application, the neurons are represented as semi-transparent cubes. Cubes are an obvious choice from a rendering point of view as there are less vertices in a cube than in a sphere. The reason the cube is semi-transparent is to enable the user to see the neuronal firing pattern. The focus of the visualization is observing the swimming pattern; the user has the ability to inspect the neurons up close in order to observe firing position of the neurons and understand their role in a macro level perspective. The cube's semi-transparency allows us to put the focus on the visual spikes rather than the neural network. An opaque visual spike will travel on a neural network of semi-transparent elements allowing us to observe the pattern created by the firing neurons.

One of the application requirements was for the user to see neural network at both Microscopic and Macroscopic level. The challenge was to be able to display 1,500 neurons and over 80,000 axons in one view while maintaining a $75 \mathrm{fps}$ (frame per second). As recommended by (Kolasinski, 1995), matching the HMD's refresh rate minimises the simulator sickness effect.

In order to have a steady frame rate of $75 \mathrm{fps}$, the rendering of the axons is a set of lines. The lines use a custom mesh for each axon, in order to minimise the number of vertices in the scene. For every point declared in the original data a set of 2 vertices has been created with a distance between vertices equal to the width of the line 0.1 Unity units (Results of the rendering tests suggested this value as it gives user the ability to see individual lines up close and at the same time to see the lines as part of the bigger neural network system). For every data point, two vertices are being generated that are connected to the next two vertices forming two triangles that in turn form a quadrilateral (quad) (Please see Fig. 8.).

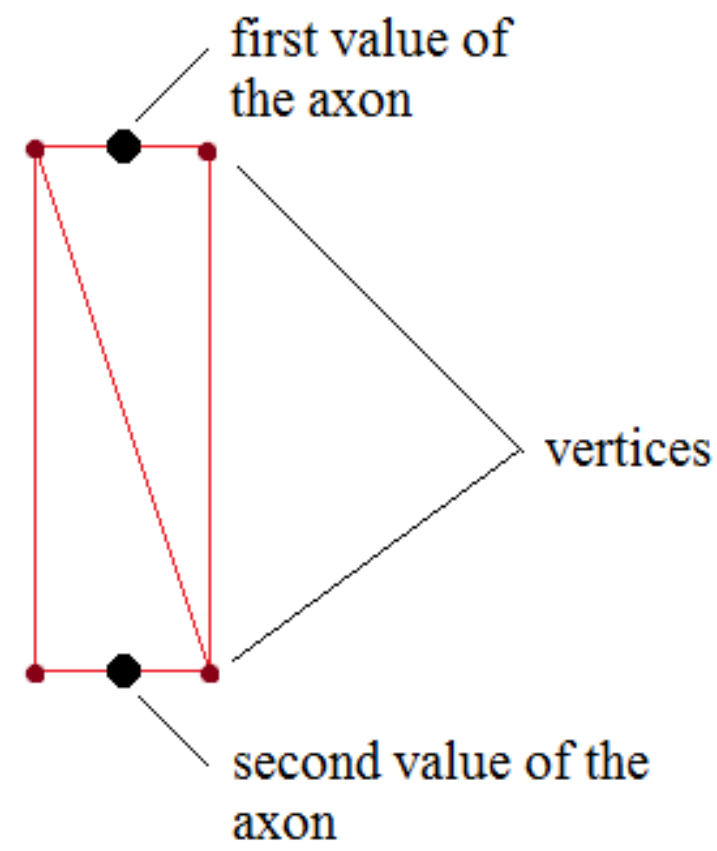

Fig. 8 The process used to generate the line that represents the axons. For every point on the axon, two vertices have been generated. Every set of two vertices are being joined to the next two vertices creating rendering triangles, which in turn form a quad.

One of the issues with rendering lines in a three-dimensional environment is from certain points of view it is 
difficult to see the shape of the line. In order to minimise this problem, each line is rotated to face the central axis of the cylinder. Non-coincidental, central axis of the cylinder is one of the best places to be in order to observe the swimming pattern. If the user places the virtual character inside the tadpole's spinal cord, around the central axis, all the rendering lines are facing the user.

To optimize the axon rendering further a "chunking system" has been created which enables culling of parts of the rendered neural network that are not in the frustum view of the camera. A chunk is an optimal size submesh that will be culled by the rendering system if not in view. All the lines that have been generated for the axons, were combined into a mesh. Dividing the cylinder with the custom chunking systems, optimized it for Unity's internal rendering system that culls any objects that are not inside the viewing frustum.

The spiking neurons are being represented by a sphere that appears at the location of the neuron. This sphere travels along the axon of that particular neuron until it reaches the final point on the axon. Reaching the end of the axon, after a short delay, the visual spiking object is disabled. Several academic writings (McIntire \& Liggett, 2014), (Fielder et al., 1996), (McIntire et al., 2014) suggest that stereoscopic effect used in VR is beneficial for understanding shape and structure inside a virtual space. Considering the poor resolution of the Oculus HMD and the fact that stereoscopic effect breaks down for elements in the distance, additional techniques to highlight the structure and its shape are needed.

(McIntire \& Liggett, 2014), (Ware, 2012a) suggest "structure from motion". Structure from motion allows the user to perceive both kinetic and parallax depth which in turn builds a better overall picture of the virtual environment around them. In order to enhance both kinetic and parallax depth in the visualization, a rendered trail to the spikes has been added. The spike will render a trail that will slowly fade out after 0.3 seconds in order to reveal a path that the spike took along the axon. The combination of spikes leaving a trail will build a visual "kinetic map" that allows the user to perceive a visual pattern of the firing neurons, leading to alternate swimming pattern becoming observable. Besides the structure from motion, a proximity luminance contrast covariance (also known as atmospheric depth) has been added as suggested by (Dosher et al., 1986). This commonly known as "fog" and has the effect of reducing the contrast of any objects in the distance (where the stereoscopic effect breaks down) enhancing user's sense of depth.

For the visual representation of the neurons, axons and spikes, 7 different colors which have been used. These colors are picked based on the previous publications of experimental and modelling data. RGB codes are: RB $(255,210,50)$; dlc - $(255,0,0)$; aIN $(70,70,180)$; cIN - $(0,170,220)$; dIN - $(150,80,30)$; mn - $(0,150,60)$; dla - $(255,170,140)$.

\subsection{Virtual environment - immersion and orientation}

\section{Immersion}

One of the Tadpole VR's main challenges was to enable the user to see not only the individual neurons firing but also the overall firing pattern of the neural network. It was crucial to have an intuitive and non-jarring experience when the user moves from Microscopic view to Macroscopic view and vice versa. In order to achieve this, a balance needs to be struck between the size of the character and the size of the neural network. As a reference, if one unit in Unity3D environment is the equivalent to one meter in real world, the character is 2-meter tall and the tadpole's neural network has a relative length of 1,600 meters. This difference in size allows the user to closely inspect individually firing neurons while at the same time, making it possible to see the overall shape of the firing pattern, when the user moves away from the neural network. This happens organically, and we have witnessed intuitive positioning by the users, without any external prompts.

The relatively big difference in size between the character and the neural network, especially in large open virtual environments makes perception of movement difficult sometimes. This typically happens when the user is exploring the Macroscopic view, away from the neural network. According to (Vishton \& Cutting, 1995) relative displacement of objects over time is essential when perceiving movement in a virtual environment. A limit on the number of three-dimensional objects has to be imposed due to the huge number of neurons and their connection. As a result, a set of three-dimensional bones, that represent the tadpole' spinal cord, has been placed around the neural network. These bones are semi-transparent and are relatively large in comparison to the neurons and allows the user to perceive movement when travelling through large open space, inside the virtual environment (See Fig. 9 , left side). For small changes in position and orientation, floating dust particles have been added that allows the user to perceive relative displacement over time, at very slow speeds.

All these elements contribute greatly to the user's immersion in the virtual environment and while sound plays 
a crucial role in immersing a user in any VR application, it is no different in this one. Every neuronal spike travelling along the axon path has a sound attached to it. The sound has a three-dimensional property for its volume intensity, the closer the user, the louder the sound. This created a unique audio effect where the user could hear the approaching spikes and once the spikes went past, the sound intensity diminished. The spiking sound makes the user feel part of the firing neural network, rather than observing it from a detached point of view. Majority of the users had complementary comments on how immersive the virtual environment feels.

\section{Orientation in virtual environment}

In any virtual environment, orientation is key in enabling the user to build a mental model of the surroundings from a self-centric point of view. This becomes increasingly difficult when the virtual environment does not have any significant unique visual features that can be used as visual anchors. In real world, gravity is used as way to give us a sense of up and down. Feeling the effect of gravity combined with having a sense of how objects are being affected by gravity, it is relatively easy to know where up or down is. In a virtual environment, the user cannot feel the effects of gravity. In the VR application, due to rendering constraints mentioned earlier there are no visual objects to simulate the effects of gravity. In their paper, (Nemire et al., 1994) suggest that linear perspective can provide a strong cue of the floor location. In the Tadpole VR, a plane with a linear grid texture (See Fig. 9, right side) is used in order to give the user cues for where the floor is and help the user identify the up and down direction of the world. Limiting the character's rotation to the vertical axis (forcing the user to always be in an upright position), the user sense of up and down is enhanced further.
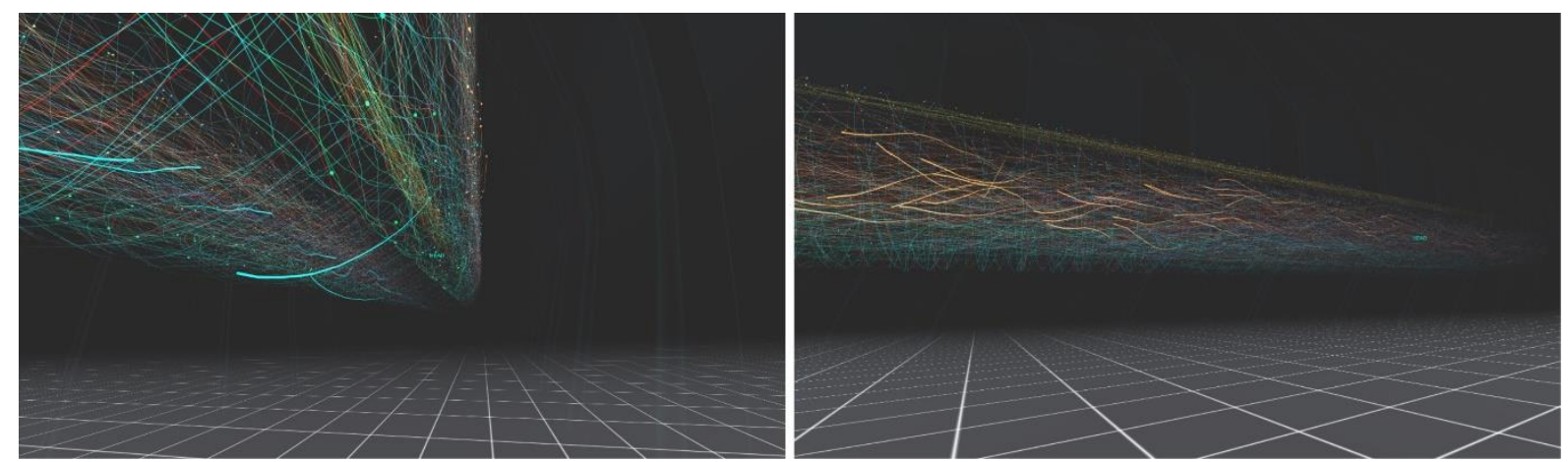

Fig. 9 Image taken from under the neural network. The see-through "bones" are visible on the back background, they help the user with navigation and orientation. Right side: Wide angle shot of the neural network, linear perspective given by the floor helps user with the sense of depth and distance

In Section 3.2 "Top down design" we discussed about Goals location and how the user is being guided with two different sets of arrows in order to move towards the goal or point of interest. This makes user's navigation and orientation easier by enabling free exploration the virtual environment. Exploring the environment freely enables the user to build a self-centric mental map of the surroundings. Due to lack of unique visual objects in virtual environment, two visual labels, with the words "head" and "tail", have been added that represent the head and tail of the tadpole's neural network. These labels act as cardinal points, so the user can use them to navigate the virtual environment. The labels are always visible regardless of how far the user is from the neural network and they are placed on a rostro-caudal direction at the head of the tadpole' spine and tail, respectively.

\section{The UsabiLITY StUdy}

A usability pilot study has been conducted to examine participant's confidence in understanding of how a neural network generates a spiking pattern, as a result of a simple touch on a side of a two-days old tadpole.

\subsection{Methodology}

\section{Participants}

Twenty computing students from University of Plymouth were recruited as participants, they were not paid. The participants age range was between 19 and 24. 19 males and 1 female were randomly chosen in a two-group design. The VR group had 11 participants; the Control group had 9 participants. None of the participants had previous knowledge about how a tadpole generates a swimming pattern inside a neural network as a result of a touch. All the participants have previously experienced VR. 


\section{Questions}

A total of 6 questions were used to capture the level of confidence the participants have in recognising the tadpole' swimming pattern and their overall understanding of how the neural network is firing. The main question was referring to how confident the participants are in observing the swimming pattern. To design the questions, (Brooke, 1996) system usability scale survey has been used. The answer for the main question regarding participant's confidence in observing the swimming pattern used the five-point Likert scale as a model. The design of the answers has four possible answers No Clue, Not Confident, Confident, Very Confident. The interpretation of the answers can be a positive or a negative sentiment. No Clue or Not Confident is considered as not confident in observing the swimming pattern (negative sentiment) while Confident and Very Confident is considered as confident in observing the swimming pattern (positive sentiment).

The design of the remaining questions was to test participant's ability to observe the location of a set of neurons inside the neural network or to describe their role in the firing sequence. These were secondary questions and they give additional information on how much understanding the participants gain from using the VR application.

\section{Approach}

Both groups were presented with an information sheet that describes how the 2-day old tadpole generates a swimming pattern as a result of a simple touch. The information sheet was composed of a diagram and support text that explains the process in non-technical terms. The participants were given a minute to study the information sheet and a control question was asked to see how confident the participants were in observing the swimming pattern. This question was repeated at the end of the study for both the VR group and the control group.

The participants in the VR group were seated and asked to put on the VR headset and they were given 2 minutes to explore the virtual environment. The visualization started with building the neural network around the user (approximately 30 seconds) and continuing with the neuron's firing pattern. After the allocated 2 minutes, five questions were asked including repeating the initial question - how confident the participants are in observing the swimming pattern. The participants kept the HMD on in order to refer to the visualization while answering questions.

The control group was seated and given a set of 6 information sheets about neural network firing patterns and the location inside the neural network of each neuron type. All sheets were composed of diagrams and descriptive text using non-technical terms. The participants had 2 minutes to study the information sheets given. At the end of the 2 minutes, they had to answer the same five questions as the VR participants while they still have access to the information sheets throughout the questioning.

\subsection{Results}

I order to measure participants understanding of how a neural network generates a spiking pattern, we formulate the main question: "How confident are you in recognizing the alternating swimming pattern propagating along the tadpole's neural network"? We use statistical tests to analyze the data collected from VR and Control groups. For all statistical tests we use 0.1 as the critical significance level for two-tailed hypothesis.

The participants were assigned randomly for the experiment, with each participant allocated to either the VR or Control group before any data collection. As a result of the random allocation and the relatively small groups of participants we find that there is a difference between initial positive sentiments: positive sentiments of $45.5 \%$ for VR group and $11.1 \%$ for Control group (see Fig. 10, VR before and Control before). We use the Mann-Whitney U Test to find if the collected data are from populations with the same distribution. The p-value is 0.1031 and that is higher than the threshold $(0.1)$, therefore it does not contradict the null hypothesis. In conclusion, the difference in the initial sentiments between two groups is not statistically different and the groups belong to populations with the same distribution (see Fig. 11 and Table 1: VR vs Control - Before). 


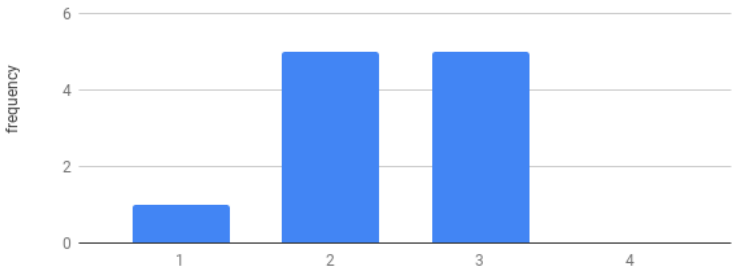

class
Control Group - before

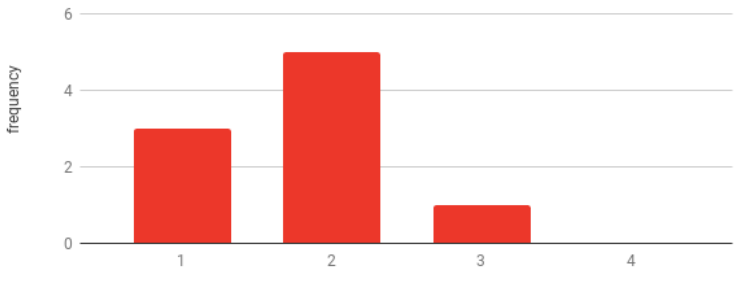

class

Fig. 11 The distribution of data collected before the use of Tadpole VR application. On the left, in blue, is the VR Group data and on the right, in red, the Control Group data.

To test the null hypothesis that data in two samples are selected from populations with the same distribution for paired observations in a "before" and "after" scenario, the Wilcoxon paired Test was used. For the Control group, the p-value is 0.2188 , therefore the data do not contradict to the null hypothesis and the distributions of positive sentiments in the Control group before and after experiment are the same. Therefore, the increase of positive sentiments in the Control group from $11.1 \%$ (before) to $33.3 \%$ (after) is not statistically significant. For the VR group the result of statistical test is different. The p-value is 0.0625 and the null hypothesis should be rejected. In the VR group the distributions of positive sentiments before and after VR application are different. Therefore, the increase of positive sentiments from $45.5 \%$ before to $81.8 \%$ after VR application is statistically significant.

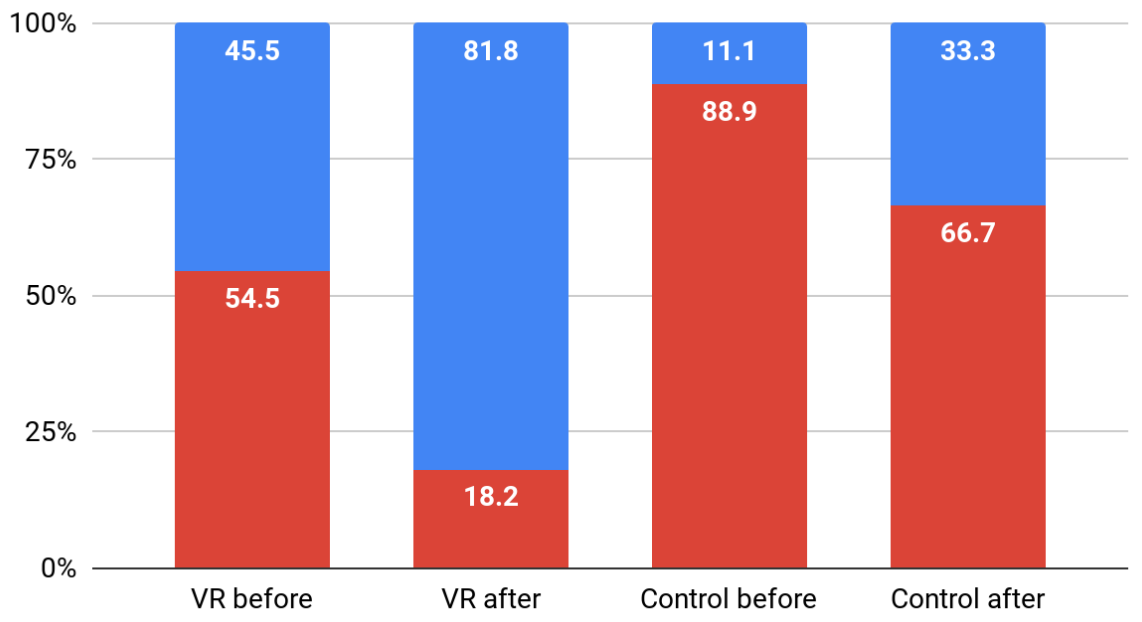

Fig. 10 Results on the level of confidence the participants have in observing the swimming pattern. A higher level of confidence for VR group after using Tadpole VR compared to the Control group

To compare results after VR application for VR and Control groups, we use the Mann-Whitney U Test. The null hypothesis is that the data are from two independent populations with the same distribution. The p-value is 0.03662 that is under the critical significance level, therefore the null hypothesis is rejected and the distributions should be considered as different. In conclusion, the increase in confidence for the VR group is significantly higher (see Fig. 12 and Table 2: VR vs Control - After). The results in the Mann-Whitney U-test are summarised in Table 1. 

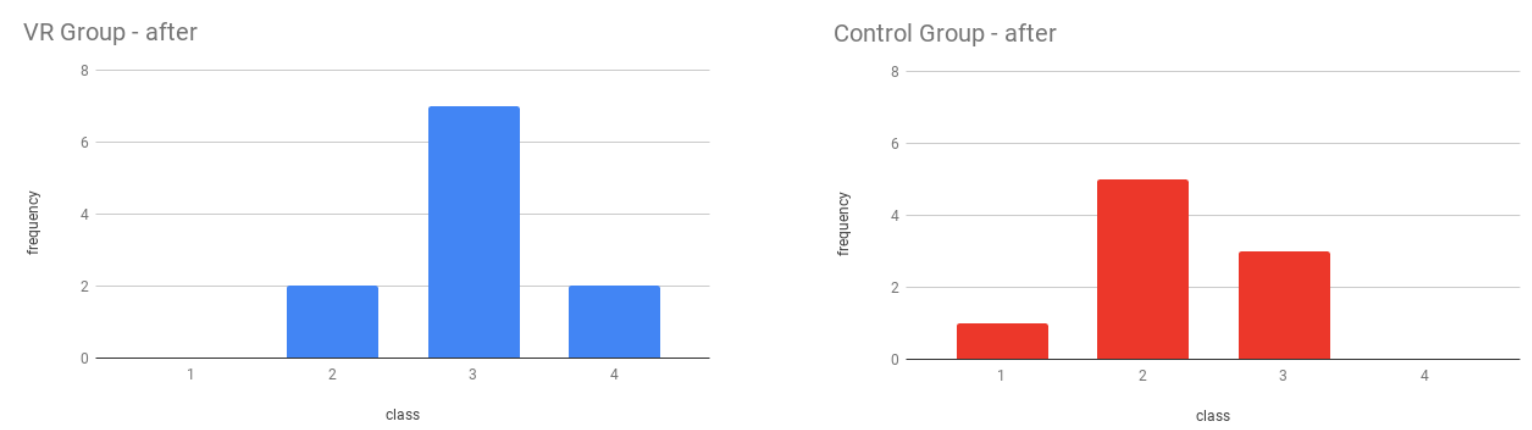

Fig. 12 The distribution of data collected after the use of Tadpole VR application. On the left, in blue, is the VR Group data and on the right, in red, the Control Group data.

\begin{tabular}{|l|c|c|}
\hline \multicolumn{1}{|c|}{ Mann-Whitney U-Test } & VR vs Control - Before & VR vs Control - After \\
\hline U-value & 27.5 & 21.5 \\
\hline U-threshold & 23 & 23 \\
\hline z-score & 1.63343 & 2.08928 \\
\hline p-value & 0.1031 & 0.03662 \\
\hline
\end{tabular}

Table 1. Results of the Mann-Whitney U-test for VR group and Control group after the experiment. Question: How confident are you in recognizing the alternating swimming pattern propagating along the tadpole's neural network?

\section{Discussion}

\subsection{Usability study other findings}

In general, the neurons spatial position in the neural network was easier to spot by both groups but the spatialtemporal complexity of firing neural network remains difficult to visualize by the Control Group, especially with the high number of neurons (1500) and over 80,000 axons. This approach seems to work really well for tasks where the user needs to identify spatial-temporal firing patterns.

An interesting find in the data was around one of the questions regarding the travelling direction of the spiking commissural neurons (cIN's), blue neurons. The control group scored relatively low (11.1\%) as opposed to VR group, which scored as high as $90.9 \%$. Although the direction of travel is clearly explained, in the information sheet, a combination of text and diagrams is needed to be able to answer correctly. The increase mental load in trying to find the information affects user's concentration and most of the participants gave up if they did not find the answer quick enough. The travelling direction for the firing pattern is clearly observable in VR, with relative no mental load. The VR group has scored very high (over $80 \%$ ) accuracy as opposed to relatively low scores for the control group. This confirms that this particular approach is suitable in observing spatio-temporal patterns created by a firing neural network.

Some of the questions relate to neuron's role as part of the neural network or its spatial location in the virtual environment. For these questions, the average response accuracy is a lot closer. One question was referring to the type of neuron that has the main axon crossing from one side of the tadpole's body to the other. The responses for the accuracy values $(90.9 \%)$ were the same but there was a significant difference in the average response time ( 5.17 for VR group and 24.99 for Control group). The difference in response time could be explained by the ability of the participants in VR group to access the necessary information at a glance. Observing the various types of neurons and their axons is quite trivial inside the visualization, therefore the participants got the correct answer near instantly. The control group had to inspect carefully the information sheet and pick up the correct information. Even though the response time was longer, the control group gave the correct answers. 
Generally, the VR group did better than the Control group for all the questions with one exception. When the participants were asked to specify the location of the Rohon-Beard neurons (yellow neurons) inside the neural network, the control group did better in both Average response time and Accuracy percentage, see Fig. 13.
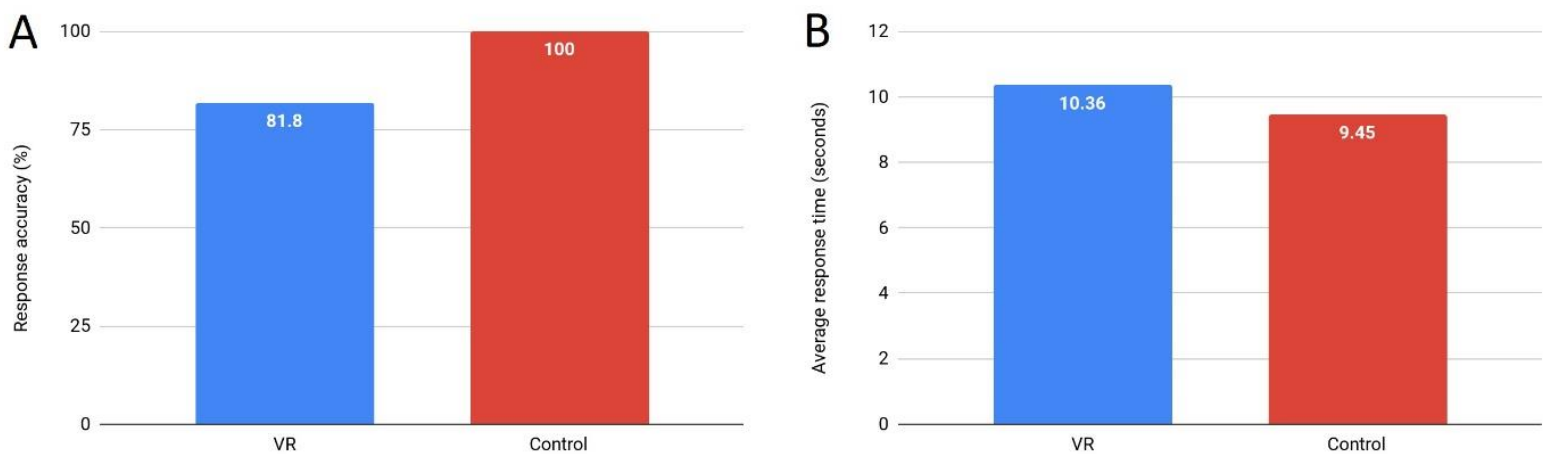

Fig. 11 (Left side) Response accuracy for both groups. VR group has performed slightly worse with an 81.8\% accuracy against the $100.0 \%$ accuracy for the Control group. (Right side) The average response time for both groups. The VR group has slower response time (10.36 seconds) than the control group (9.45 seconds).

The Control group had a faster response time due to the quick access to this information. One of the information sheet contain a printout of the side view on the neural network. There were colored bands showing in the image, so it was relatively easy to observe the location of the Rohon-Beard neurons. That explain both the accuracy of the responses for the control group and the relatively small average response time.

For VR group, we believe there are a few factors that contribute to the lower scores for both correct answers and response time. First major factor is the relative restricted field of view of the VR headset. Majority of the users were observing the neural network from inside around central axis of the cylinder shape of the neural network. With a restricted field of view (letter-box effect for HMD's) and due to the location of the Rohon-Beard (yellow) neurons, at the top of the neural network, participants needed to tilt their head up to see them. Another factor that contributed to lower scores was the combination of colors in front of the user. In the midsection of the neural network, where most of the users were looking, there are a number of different neurons: blue (cIN's), green (mn's), purple (aIN's) all cold colors. Amongst these neurons, the dIN's neurons, which have an orange color, are a warm color. For a participant, with a restricted field of view (not being able to see the yellow neurons at the top), these orange neurons look yellow in comparison with the cold color neurons around it, therefore they though the yellow neurons are located in the midsection of the neural network. We checked the data for this particular question, and it confirms this hypothesis.

\subsection{Potential Applications}

\section{Education}

Tadpole VR has great potential in a variety of fields, but mainly in knowledge dissemination (especially for non-experts), neuroscience and education. Deploying the VR application on mobile devices and using a stereoscopic viewing device similar to Google Cardboard ${ }^{5}$, hundreds of students can benefit sitting in a lecture theatre observing the complex structure of a neural network and the convoluted firing pattern of its neurons. The relative low cost of Google Cardboard makes this an attractive proposition. The intention is not to replace the teacher or the teaching material but to use it as an additional source of information, more accessible and more intuitive, minimizing the mental load.

Due to the high number of neurons and axons, an optimization process is required before the visualization is ready for mobile VR. This will be an opportunity to improve the visualization. Currently, lines represent the connection between neurons (axons). Line rendering delivers a high frame rate and works when viewed at short distances in VR. The disadvantage is lack of depth cues at long distances. According to (Ware \& Mitchell, 2008) a combination of stereoscopic effect, kinetic depth and using three-dimensional shaded tubes will lead to a greater increase in observable complex node graphs. This visualization, at its core, is a complex set of node graphs and with the latest advances in hardware and rendering technology it is possible to render all the axons as shaded

\footnotetext{
${ }^{5}$ Google Cardboard - https://vr.google.com/cardboard/
} 
tubes, improving the visual representation of the neural network.

\section{Analytical tool}

Another possible application for Tadpole VR is as an analytical tool for neuroscience experts. It has a direct application in the neuroscience field, where current progress leads to more complex mathematical and computational models. In a recent paper by (Davis et al., 2017), the axon growth model was adapted to include interaction between growing axons (fasciculation). How does such axon-axon interaction affect the resulting trajectories and produce fasciculation? A VR visualization like Tadpole VR can address these issues by enabling the experts to understand and visually study developmental processes in the nervous system while manipulating the growth parameters.

\section{Conclusion And Future Development}

In this paper, we presented a three-dimensional VR visualization of an observable swimming pattern. The swimming pattern is generated as a result of the neuronal activity in the spinal cord of a simulated two-day old tadpole. This visualization is based on a biologically realistic computational model containing approximately 1,500 neurons and 80,000 axon connections. A detailed description of the original dataset was given, together with the steps taken for preparing the data for rendering in a VR environment. This includes a top-down approach to the design of the virtual environment, based on a set of requirements. Followed up by the restrictions imposed by the bottom-up design and the compromise achieved between the application requirements and the hardware and software limitations.

Although the relatively small number of participants for the usability study does not give us a definite answer, there is encouraging evidence that this approach allows participants to observe a complex firing pattern inside a virtual world. Overall, there is a clear increase in participant's confidence in observing the swimming pattern, with participants doing especially well in spotting the spatio-temporal behavior of the firing neurons.

Although a formal study, on the effectiveness of the VR application, has not been run, informal discussion have been taking place with participants at a range of conferences and public events: Neuroinformatics 2016 International conference ${ }^{6}$, UK Robotics $\mathrm{Week}^{7}$ and $\mathrm{CogNovo}^{8}$ research seminars. In total, over 70 participants experienced the visualization and we received positive feedback regarding the immersive element of the application. In the future, we would like to run a usability study on the effectiveness of the VR application in order to assess its potential.

Future work could introduce a more intuitive set of controls, with 6 degrees of freedom, similar to Oculus Touch $^{9}$ controller. The controllers will track user's hands inside the virtual environment. This opens up a new set of possibilities with the users being able to interact with the three-dimensional objects in a more intuitive way. Interaction or selection of the individual neurons will be possible, including isolating types of neurons or watching the computational model responding to changes in growth parameters inside the virtual environment. This will pave the way for neuroscientists to use the application as an analytical tool.

The development of this application and the pilot study did answer some questions, but more questions remain. Is there a better way to differentiate between different types of neurons other than color? In Tadpole VR, the seven different types of neurons are being differentiate by color; shape, size or luminosity could play a role in differentiating between neurons. At this point in time the answer is not certain, more research is needed.

This paper demonstrated that spatial layout plays an important role in understanding the functionality of a neural network. What about the spatial layout for an abstract data set that does not have a specific location? What is the best approach to distribute an abstract, complex data set in an immersive virtual environment, for easy understanding?

These questions have not been fully answered yet and the field of information visualization can benefit from more research into immersive VR visualizations. To inform future development for this type of VR applications further investigation is needed.

\footnotetext{
${ }^{6}$ Neuroinformatics - https://www.frontiersin.org/events/Neuroinformatics_2016/3552

${ }^{7}$ UK Robotics Week - https://www.plymouth.ac.uk/whats-on/uk-robotics-week-at-plymouth-university

${ }^{8}$ CogNovo Research Seminars - https://www.plymouth.ac.uk/whats-on/cognovo-research-seminars

${ }^{9}$ Oculus Touch - https://www.oculus.com/rift/accessories/?locale=en_GB
} 


\section{REFERENCES}

Armstong J D and Van Hemert J I (2009) Towards a virtual fly brain, Philosophical Transactions of the Royal Society A: Mathematical, Physical and Engineering Sciences. Royal Society, 367(1896), pp. 2387-2397. doi: 10.1098/rsta.2008.0308.

Arsiwalla X., Zucca R., Betella A., Martinez E., Dalmazzo D., Omedas P., Deco G., Vershure P. (2015) Network dynamics with BrainX3: a large-scale simulation of the brin network with real-time interaction. Front Neuroinform, 9:2:1-14

Borisyuk R, Al Azad A K, Conte D, Roberts A \& Soffe S R (2014) A developmental approach to predicting neuronal connectivity from small biological datasets: a gradient-based neuron growth model. PloS One, 9(2), e89461. https://doi.org/10.1371/journal.pone.0089461

Brooke J. (1996) SUS-A quick and dirty usability scale. Usability evaluation in industry, 189(194), pp.4-7.

Bruckner S, Solteszova V, Groller E, Hladuvka J, Buhler K, Yu J Y, et al., (2009) Braingazer-visual queries for neurobiology research Visualization and Computer Graphics, IEEE Transactions on, vol. 15, pp. 1497-1504.

Bryson S, (1995) Approaches to the successful design and implementation of VR applications. In: R.A. Earnshaw, J.A. Vince and H. Jones (eds) Virtual Reality Applications. Academic Press Inc., San Diego, pp. 3-15.

Davis O, Merrison-Hort R, Soffe S R \& Borisyuk R (2017) Studying the role of axon fasciculation during development in a computational model of the Xenopus tadpole spinal cord. Sci. Rep., vol. 7, no. 1, p. 13551

Dosher B A, Sperling G \& Wurst S A (1986) Tradeoffs between stereopsis and proximity luminance covariance as determinants of perceived 3D structure. Vision research, 26(6), pp.973-90. Available at: http://www.ncbi.nlm.nih.gov/pubmed/3750879. Accessed 5 January 2018

Esmaeili H \& Thwaites H (2016) Virtual photogrammetry. In 2016 22nd International Conference on Virtual System \& Multimedia (VSMM)(pp.1 - 6). IEEE. https://doi.org/10.1109/VSMM.2016.7863153

Ferrario A, Merrison-Hort R, Soffe S R \& Borisyuk R (2018) Structural and functional properties of a probabilistic model of neuronal connectivity in a simple locomotor network. eLife, 7. Available at: https://elifesciences.org/articles/33281 Accessed February 26, 2019

Fielder A R, Moseley M J \& London M J M (1996) Does stereopsis matter in humans' eye, 10(2), pp.233-238. Available at: http://www.nature.com/doifinder/10.1038/eye.1996.51. Accessed 1 January 2017

Hubbell M and Kepner J (2012) Large scale network situational awareness via 3D gaming technology, 2012 IEEE Conference on High Performance Extreme Computing, pp. 1-5.

Kapri A V R, Rick, Tobias P, Tobias C, Diesmann M \& Kuhlen T (2011) Towards the visualization of spiking neurons in virtual reality. Studies in health technology and informatics, 163, pp.685-7.

http://www.ncbi.nlm.nih.gov/pubmed/21335880. Accessed 5 January 2018

Kasabov N, Scott NM, Tu E, Marks S, Sengupta N, Capecci E, Othman M, Doborjeh MG, Murli N, Hartono R, EspinosaRamos J, Zhou L, Alvi FB, Wang G, Taylor D, Feigin V, Gulyaev S, Mahmoud M, Hou ZG, Yang J (2016) Evolving spatiotemporal data machines based on the neucube neuromorphic framework: Design methodology and selected applications. Neural Networks 78:1-14

Kolasinski EM (1995) Simulator Sickness in Virtual Environments. Tech. rep., Defense Technical Information Center, URL www.dtic.mil/cgi-bin/ GetTRDoc?AD=ADA295861

Lee, S. et al., 2003. Evaluation of pointing techniques for ray casting selection in virtual environments. IN THIRD INTERNATIONAL CONFERENCE ON VIRTUAL REALITY AND ITS APPLICATION IN INDUSTRY, pp.38--44. Available at: http://citeseerx.ist.psu.edu/viewdoc/summary?doi=10.1.1.122.3662 [Accessed January 7, 2018].

Lin C Y, Tsai K L, Wang S C, Hsieh C H, Chang H M, and Chiang A S, (2011) The Neuron Navigator: Exploring the information pathway through the neural maze," in Pacific Visualization Symposium (PacificVis), 
2011 IEEE, pp. 35-42.

Marder E, Goeritz ML \& Otopalik AG (2015) Robust circuit rhythms in small circuits arise from variable circuit components and mechanisms. Current opinion in neurobiology, 31, pp.156-63. Available at: http://www.ncbi.nlm.nih.gov/pubmed/25460072. Accessed March 10, 2019

Marks S, Estevez J E \& Connor A M (2014) Towards the Holodeck: Fully Immersive Virtual Reality Visualisation of Scientific and Engineering Data. Proceedings of the 29th International Conference on Image and Vision Computing New Zealand - IVCNZ'14, pp.42-47. http://arxiv.org/abs/1604.05797. Accessed 5 January 2018

Marks S (2017) Immersive visualisation of 3-dimensional spiking neural networks. Evolving Systems, 8(3), pp.193-201

McIntire J P, Havig P R. \& Geiselman E E (2014) Stereoscopic 3D displays and human performance: A comprehensive review. Displays, 35(1), pp.18-26. https://www.sciencedirect.com/science/article/pii/S0141938213000929. Accessed 7 January 2018

McIntire J P \& Liggett K K (2014) The (possible) utility of stereoscopic 3D displays for information visualization: The good, the bad, and the ugly. In 2014 IEEE VIS International Workshop on 3DVis (3DVis). IEEE, pp. 1-9. Available at: http://ieeexplore.ieee.org/document/7160093/. Accessed 6 April 2017

Moran A et al. (2015) Improving Big Data visual analytics with interactive virtual reality. In 2015 IEEE High Performance Extreme Computing Con-ference (HPEC). IEEE, pp. 1-6. Available at:

http://ieeexplore.ieee.org/document/7322473/ [Accessed January 6, 2017].

Nemire K, Jacoby R H \& Ellis S R (1994) Simulation Fidelity of a Virtual Environment Display. Human Factors: The Journal of the Human Factors and Ergonomics Society, 36(1), pp.79-93.

http://journals.sagepub.com/doi/10.1177/001872089403600105. Accessed 5 January 2018

Roberts A, Li W C and Soffe S R (2010) How neurons generate behaviour in a hatchling amphibian tadpole: an outline, Front. Behav. Neurosci., vol. 4, p. 16

Roberts A, Conte D, Hull M, Merrison-Hort R, al Azad A K, Buhl E, Borisyuk R \& Soffe S R (2014) Can simple rules control development of a pioneer vertebrate neuronal network generating behavior? The Journal of Neuroscience:The Official Journal of the Society for Neuroscience, 34(2), 608-21. https://doi.org/10.1523/JNEUROSCI.3248-13.

Sherif T, Kassis N, Rousseau ME, Adalat R, Evans AC (2015) BrainBrowser: distributed, web-based neurological data visualization. Frontiers in Neuroin- formatics 8:89, DOI 10.3389/fninf.2014.00089, URL http://journal. frontiersin.org/article/10.3389/fninf.2014.00089/full

Vishton P M \& Cutting J E (1995) Wayfinding, displacements, and mental maps: velocity fields are not typically used to determine one's aimpoint. Journal of experimental psychology. Human perception and performance, 21(5), pp.978-95. http://www.ncbi.nlm.nih.gov/pubmed/7595249. Accessed 5 January 2018

Ware C (2012) Information visualization : perception for design, Morgan Kaufmann.

http://www.sciencedirect.com/science/book/9780123814647 pp. 280, pp. 256. Accessed 5 January 2018

Ware C (2012) Information visualization : perception for design, Morgan Kaufmann.

http://www.sciencedirect.com/science/book/9780123814647, pp. 358. Accessed 5 January 2018

Ware C \& Mitchell P (2008) Visualizing graphs in three dimensions. ACM Transactions on Applied Perception, 5(1), pp.115. http://portal.acm.org/citation.cfm?doid=1279640.1279642. Accessed 21 April 2017

Xia M, Wang J, He Y (2013) BrainNet Viewer: A Network Visualization Tool for Human Brain Connectomics. PLOSONE 8(7):e68,910, DOI 10.1371/journal.pone. 0068910, URL http://journals.plos.org/plosone/article?id= 10.1371/journal.pone.0068910

Yao R, Heath T, Davies A, Forsyth T, Mitchell N and Hoberman P (2015) Oculus Best Practices. Available at: https://static.oculus.com/documentation/pdfs/intro-vr/latest/bp.pdf [Accessed January 7, 2018]. 